\title{
Non-Poisson distribution of the time distances between two consecutive clusters of earthquakes
}

\author{
Luigi Palatella ${ }^{\mathrm{a}}$, Paolo Allegrini ${ }^{\mathrm{b}}$, Paolo Grigolini ${ }^{\mathrm{a}, \mathrm{c}, \mathrm{d}}$, \\ Vito Latora $^{\mathrm{e}}$, Mirko S. Mega ${ }^{\mathrm{a}}$, Andrea Rapisarda ${ }^{\mathrm{e}}$, \\ Sergio Vinciguerra ${ }^{\mathrm{e}}$ \\ ${ }^{a}$ INFM - Dipartimento di Fisica dell'Università di Pisa \\ via Buonarroti 2, 56127 Pisa, Italy \\ ${ }^{\mathrm{b}}$ Istituto di Linguistica Computazionale del CNR, \\ Via Moruzzi 1, 56124, Pisa, Italy \\ ${ }^{\mathrm{c}}$ Istituto dei Processi Chimico Fisici del CNR, \\ Via G. Moruzzi 1,56124 Pisa, Italy \\ ${ }^{\mathrm{d}}$ Center for Nonlinear Science, University of North Texas,P.O. Box 311427, \\ Denton, Texas 76203-1427 \\ ${ }^{\mathrm{e}}$ Dipartimento di Fisica e Astronomia, Università di Catania, and INFN, \\ Via S. Sofia 64, 95123 Catania, Italy
}

\begin{abstract}
With the help of the Diffusion Entropy technique we show the non-Poisson statistics of the distances between consecutive Omori's swarms of earthquakes. We give an analytical proof of the numerical results of an earlier paper [Mega et al., Phys. Rev. Lett. 90 (2003) 188501].
\end{abstract}

Key words: Earthquakes, time-series analysis, anomalous scaling PACS: 91.30.Dk,05.45.Tp,05.40.Fb

The model usually adopted to describe the time distribution of earthquakes is the Generalized Poisson (GP) model $[1,2,3,4,5]$. The GP model assumes that the earthquakes are grouped into temporal clusters of events and these clusters are uncorrelated, and, therefore, completely unpredictable: The clusters are supposed to be distributed at random in time and therefore the time intervals between one cluster and the next one follow a Poisson distribution. The intracluster earthquakes are in fact correlated as it is expressed by the Omori's law [6,7], an empirical law stating that the main shock, i.e. the highest magnitude 
earthquake of the cluster, occurring at time $t_{0}$ is followed by a swarm of triggered earthquakes (after shocks) whose number (or frequency) $n(t)$ decays in time as a power law, $n(t) \propto\left(t-t_{0}\right)^{-p}$, with the exponent $p$ being very close to 1 . If we denote with the symbol $\tau$ the time intervals between one earthquake and the next, then right after a main shock, a short value of $\tau$ is followed with a large probability by another short value. For the same reason, far from a main shock and prior to the next one, a long value of $\tau$ is followed by another long value of $\tau$. This implies that the correlation function $\left\langle\left(\tau_{i}-\langle\tau\rangle\right)\left(\tau_{j}-\langle\tau\rangle\right)\right\rangle$ is not zero for $i \neq j$ and that it survives for all pairs of seismic events in between two consecutive unpredictable shocks. Omori's law also implies [8] that the distribution of $\tau$, is a power law $\psi(\tau) \propto \tau^{-p}$. This espression is valid in the time regime inside a swarm, and it is then truncated by a sharp cutoff caused by the arrival of the next swarm.

The catalog we have studied covers the period 1976-2002 in the region of Southern California spanning $20^{\circ} \mathrm{N}-45^{0} \mathrm{~N}$ latitude and $100^{0} \mathrm{~W} 125^{\circ} \mathrm{W}$ longitude [9]. This region is crossed by the most seismogenetic part of the San Andrea fault, which accommodates by displacement the primarily strike-slip motion between the North America and the Pacific plates, producing velocities up to $47 \mathrm{~mm} / \mathrm{yr}$ [10]. The total number of recorded earthquakes in the catalog is 383687 .

Herein we disprove the GP model, providing evidence for the non-Poisson statistics of inter-cluster times, by applying to the mentioned catalog the Diffusion Entropy (DE) technique [11]. Here we discuss with analytical arguments some issues that in an earlier paper [12] we have examined by means of a numerical treatment. As in the GP model, we assume that each cluster starts with an unpredictable triggering event (it may or may not be the main-shock). The distance between the $i$-th and the $i+1$-th cluster is therefore the time distance between such events, which we indicate as $\tau_{i}^{[m]}$, obeying the noncorrelation property, $\left\langle\tau_{i}^{[m]} \tau_{j}^{[m]}\right\rangle=\left\langle\tau^{[m]}\right\rangle^{2}$ if $i \neq j$. The superscript $m$ stands for main-shock, but we actually need not to make the assumption that the triggering event is a large earthquake. As we shall see, the DE measures statistical properties of events with no need of identifying them.

Let us recall the definition of the DE functional $S(t)$ as the Shannon entropy of $p(y, t)$, the probability distribution to observe a fixed number of seismic events $y$ in a given time interval [11]. This observation is equivalent to observing the spreading of a number of walkers making one step forward at each time where an event is met. Hence the term "Diffusion Entropy". Different trajectories are chosen with the usual method of observing different time windows in the sequence, which is herein assumed to be stationary. Let us assume that $p(y, t)$ follows the scaling law with the form

$$
p(y, t)=\frac{1}{t^{\delta}} F\left(\frac{y}{t^{\delta}}\right)
$$


where $\delta$ is a positive exponent and $F(x)$ is a positive and integrable function of $\mathrm{x}$. As a consequence of this assumption, after a straightforward algebra, we find that

$$
S(t)=A+\delta \ln (t)
$$

This means that the entropy of the diffusion process is a linear function of $\ln (t)$ and a measure of the slope is equivalent to the determination of the scaling parameter $\delta$.

We now show that, in the earthquake series under investigation, the principal source of entropy increase is given by the occurrence of the cluster-initiating seismic events. Let us indicate with $\phi\left(\tau^{[m]}\right)$ the probability density function (pdf) of times between clusters and with $h(x)$ the pdf of the number of earthquakes in a cluster. The function $h(x)$, usually referred to as the Pareto's law of earthquakes, is known to decay as $h(x) \simeq 1 / x^{\alpha+1}$, where $\alpha$ is a positive number. In the literature an exponent for clusters size distribution $\alpha$ ranging from $\alpha=1.25$ [13] to 3 [4] is reported. On the other hand, we assume for $\phi\left(\tau^{[m]}\right)$, which is exponential in the GP model, a form $\phi\left(\tau^{[m]}\right) \simeq\left(1 / \tau^{[m]}\right)^{\mu}$. We also assume that $2 \leq \mu<3$. As it will become clear in the next paragraphs, beyond the upper limit it is impossible for the DE to distinguish between an inverse-power law and an exponential; for $\mu<2$, on the other hand, the signal cannot be stationary [11]. The connection between $x$ and the early defined variable $y$, over which the $\mathrm{DE}$ is calculated, is

$$
y(t)=\sum_{i=1}^{z(t)} x_{i}
$$

where the sum is carried over different clusters, $x_{i}$ is the number of shocks in the $i$-th cluster and $z(t)$ is the number of clusters in the same time window of length $t$ considered for $y$.

Let us call $\widehat{\phi}(s)$ the Laplace transform of $\phi\left(\tau^{[m]}\right)$ and $\widehat{h}(k)$ the Fourier transform of $h(x)$. Suppose now that the time duration of a cluster is negligible with respect to the mean time distance $\left\langle\tau^{[m]}\right\rangle$. In this case we can use directly a Continuous Time Random Walk (CTRW) formalism to calculate the probability $p(y, t)$ that a random walker, moving of a quantity $x$ at the time at which there is a cluster of size $x$ (and resting otherwise), is at position $y$ after a time $t$. For the Fourier-Laplace transform of $p(y, t)$ we will have, from the theory of CTRW $[14,15]$,

$$
\widehat{p}(k, s)=\frac{1-\widehat{\phi}(s)}{s} \frac{1}{1-\widehat{\phi}(s) \widehat{h}(k)} .
$$


To obtain the asymptotical behavior we write, if $2<\mu<3$,

$$
\widehat{\phi}(s) \simeq 1-\left\langle\tau^{[m]}\right\rangle s+c s^{\gamma}
$$

where $\gamma \equiv \mu-1$. Eq.(4) becomes

$$
\widehat{p}(k, s) \simeq \frac{\left\langle\tau^{[m]}\right\rangle}{1-\widehat{h}(k)+s\left\langle\tau^{[m]}\right\rangle \widehat{h}(k)-c \widehat{h}(k) s^{\gamma}} .
$$

Considering that

$$
\widehat{h}(k) \simeq 1+i k\langle x\rangle+b k^{\alpha}
$$

if $1<\alpha<2$, we see that Eq. (6) leads to a ballistic scaling in the laboratory reference frame. The DE is insensitive to drifts, so we assume that Eq. (1) is fulfilled in a "detrended" moving reference frame, namely, where the position of the walker $y$ fulfills the condition $\langle y(t)\rangle=0$ at each time $t$. To obtain $\widehat{p}(k, s)$ in such a moving reference frame we have to perform the following substitution

$$
s \rightarrow s+i k \frac{\langle x\rangle}{\left\langle\tau^{[m]}\right\rangle}
$$

After that, we apply the diffusive limit $k \gg s$ obtaining

$$
\widehat{p}(k, s) \simeq \frac{\left\langle\tau^{[m]}\right\rangle}{s\left\langle\tau^{[m]}\right\rangle-b k^{\alpha}-c\left(i\langle x\rangle /\left\langle\tau^{[m]}\right\rangle\right)^{\gamma} k^{\gamma}},
$$

thus proving that the most anomalous, namely, the smallest between the two exponents, either $\alpha+1$ or $\mu$, determines the asymptotic scaling according to the prescription $\delta=1 / \alpha$ or $\delta=1 /(\mu-1)$, respectively.

In Fig. 1 we report the results of the DE method. In full circles we plot the entropy $S(t)$ as a function of time when all the seismic events of the catalog are considered (independently of their magnitude $M$ ). A fit in the linear region gives a value of the scaling parameter $\delta=0.94$. We next consider (open symbols in Fig. 1) only the earthquakes with magnitude larger than a fixed value $\bar{M}=2,3,4$. We see that, regardless of the value of the threshold $\bar{M}$ adopted, the function $S(t)$ is characterized by the same long-time behavior with the same slope. This indicates that we are observing a property of the time location of the main earthquakes. Moreover, if the value of $\delta$ were due to $h(x)$ we should observe at most $\delta=1 / \alpha=1 / 1.25=0.80$. This leads us to conclude that the asymptotic form (and scaling) of $p(y, t)$ is determined by the probability $p(z, t)$ of finding $z$ unpredictable events in a time window 


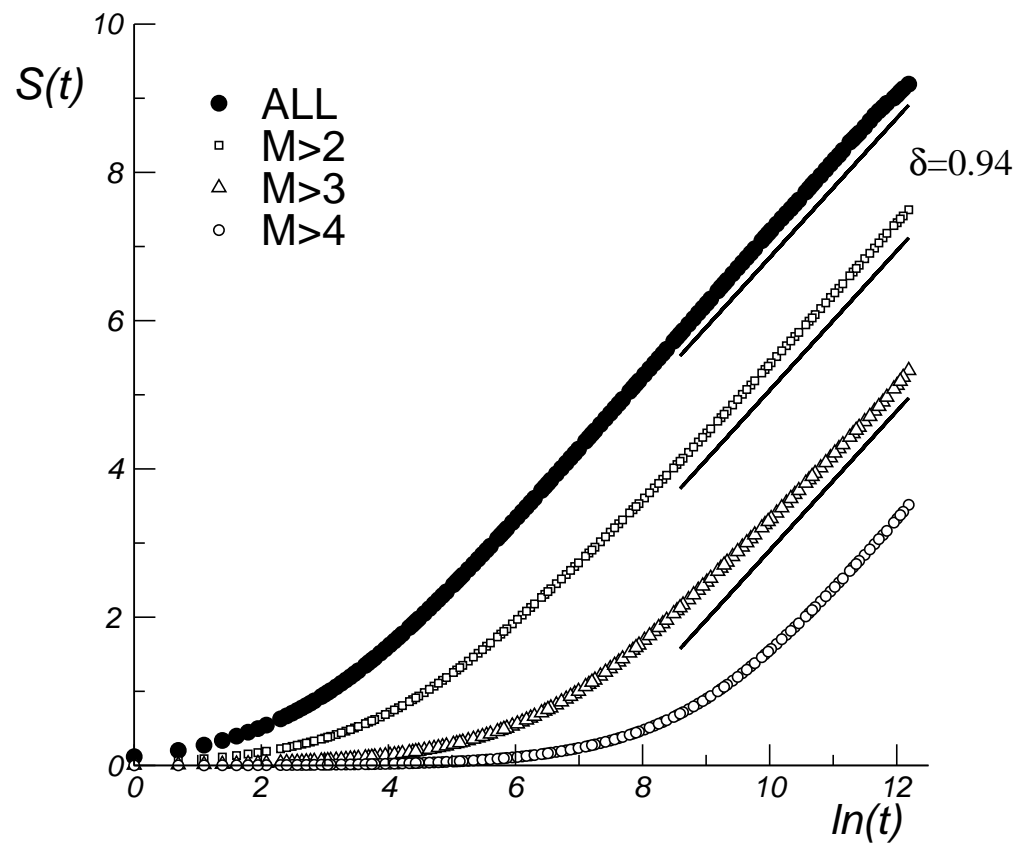

Fig. 1. The Shannon entropy $S(t)$ of the diffusion process as a function of the logarithm of time, expressed in minutes. From top to bottom, the curves refer to all events (full circles) and to events with threshold $\bar{M}=2,3,4$ (open symbols). The straight lines have the slope $\delta=0.94$.

of duration $t$. This is in turn determined by a $\phi\left(\tau^{[m]}\right)$ decaying as an inverse power law with exponent $\mu=2.06$. Using the theory of Ref. [11] we also determine the form of $p(z, t)$, which is well approximated by an asymmetric Lévy distribution with Lévy index $\mu-1$.

In conclusion, in this paper we have studied the statistical properties of earthquakes time distribution. Inter-cluster distances obey an inverse power law prescription $\phi\left(\tau^{[m]}\right) \propto\left(\tau^{[m]}\right)^{-\mu}$ with $\mu=2.06 \pm 0.01$ thus ruling out the GP model. The method proposed is based on the fact that the asymptotic properties of diffusion process generated by the seismic events are scarsely sensitive to the memory stemming from the Omori's law. They are, on the contrary, sensitive to the anomalous statistics generated by the non-Poisson nature of the time distance between two consecutive large earthquakes. This non-Poisson behavior reflects, in our opinion, the cooperative behavior of the geological processes triggering the main shock, and consequently, to some extent, some sort of predictability. The emergenge of this possibility will be investigated in the future.

As a final remark, we recall that throughout our analysis we made the reasonable hypothesis that the series is stationary. On a formal ground, a scaling value $\delta \sim 1$ is compatible with a nonstationary process. These nonstationary contributions may have different orgins. The most trivial is the lack of statistics: we may have for instance, a small number of Omori's swarms, but very 
extended in time. For this reason we repeated the analysis using only portions of the series, obtaining the same results. Another, more interesting, source of non-stationarity could be a strong correlation among the series of the triggering processes, namely the condition $\left\langle\tau_{i}^{[m]} \tau_{j}^{[m]}\right\rangle \neq\left\langle\tau^{[m]}\right\rangle^{2}$. It is interesting to notice that also this condition might reflect a form of predictability to assess through a properly tailored form of statistical analysis of time series. We leave this as a subject of further investigation.

PG thankfully acknowledges the Army Research Office for financial support through Grant DAAD19-02-0037.

\section{References}

[1] S. Shlien, M.N. Toksoz, Bull. Seism. Soc. Am. 60 (1970) 1765.

[2] J.K. Gardner and L. Knopoff, Bull. Seism. Soc. Am. 64 (1974) 1363.

[3] P. Gasperini, F. Mulargia, Bull. Seism. Soc. Am. 79 (1989) 973.

[4] R. Console, M. Murru, J. Geophys. Res. 106 (2001) 8699.

[5] C. Godano and V. Caruso, Geophys. J. Int. 121 (1995) 385.

[6] F. Omori, J. College Sci. Imper. Univ. Tokyo 7 (1895) 111.

[7] T. Utsu, Geophys. Mag. 30 (1961) 521.

[8] P. Bak, K. Christensen, L. Danon and T. Scanlon, Phys. Rev. Lett 88 (2002) 178501.

[9] The catalog has been downloaded from the Southern California Earthquake Data Centre http://www.scecdc.scec.org/ftp/catalogs/SCSN/

[10] D. L. Turcotte and G. Schubert, Geodynamics, Cambridge University Press 2002.

[11] P. Grigolini, L. Palatella, G.Raffaelli, Fractals 9 (2001) 439.

[12] M. S. Mega, P. Allegrini, P. Grigolini, V. Latora, L. Palatella, A. Rapisarda, S. Vinciguerra, Phys. Rev. Lett. 90 (2003) 188501.

[13] A. Helmstetter Phys. Rev. Lett. 91 (2003) 058501.

[14] E.W. Montroll, G.H. Weiss, J. Math. Phys. 6 (1965) 167.

[15] L. Palatella, J. Perello, M. Montero, J. Masoliver, cond-mat/0312489. 